\title{
Dakshayani Indices on Carbon and Boron Nitride Nanotubes
}

\author{
Basavarajappa N S ${ }^{1}$, Shanmukha M C ${ }^{2, *}$ (I), Niranjan K M ${ }^{3}$, Shilpa K C ${ }^{1}$ \\ 1 Department of Mathematics and Computer Science Engg, Bapuji Institute of Engineering and Technology, Davanagere- \\ 577004, Karnataka, India \\ 2 Department of Mathematics, Jain Institute of Technology, Davanagere-577003, Karnataka, India \\ 3 Department of Mathematics, UBDT College of Engineering, Davanagere-577004, Karnataka, India \\ * Correspondence: mcshanmukha@gmail.com;
}

Scopus Author ID 57217312072

Abstract: Chemical graph theory is a discipline of graph theory deals with the study of chemical compounds using simple connected graphs. In computational chemistry, the properties of chemical compounds are determined using a numerical value called the topological index. Topological indices are widely applicable in QSAR and QSPR modeling. In this proposed work, we have used topological index like Neighborhood Dakshayani index on nanostructures called Carbon nanotube and Boron nitride nanotubes. Carbon nanotube has its various applications in air and water filtration, field emission, and biomedical applications. Boron nitride nanotubes are used in aerospace to automotive and defense to biomedical.

Keywords: Nanostructures; Neighborhood Dakshayani indices; Carbon nanotubes; Boron nitride nanotubes.

(C) 2020 by the authors. This article is an open-access article distributed under the terms and conditions of the Creative Commons Attribution (CC BY) license (https://creativecommons.org/licenses/by/4.0/).

\section{Introduction}

Graph theory is used in almost all fields of studies. Chemical graph theory deals with the discussion of chemical compounds using simple graphs. The graph $\mathrm{G}$ includes vertices and edges such that chemical compound atoms can be represented by vertices and the links between the atoms as edges. The total graphs number of edges in association with the vertices known as the degree of a vertex. The chemists use graph theory as a computing measure to determine the organization of chemical compounds since the valency of an atom is the degree of a vertex. The chemical graph theory offers the study of characteristics of chemical compounds. The topological indices are the numerical quantities used to determine the properties of chemical compounds. In the molecular graph, the vertices are denoted as atoms, and the edges are denoted by the links connecting the vertices. Chemical graph theory has its application in the development of chemical science and medical science. The mathematical chemistry offers several topological indices used in QSAR/QSPR study. For the discussion of topological indices, see [5, 6, 13, 14, 15, 17].

Nanosystem includes various organic and inorganic compounds that are responsible for their characteristics whose size varies from 1 to $100 \mathrm{~nm}$. Examples of nanostructures include nano-torus, nanotubes, etc. So far, the work has been done on neighborhood degree-based indices in $[8,9,10,11,16]$. In this proposed work, we use a few defined degree-based 
topological indices such as the first and second neighborhood Dakshayani indices, the first and second hyper neighborhood Dakshayani indices, and the minus and square neighborhood Dakshayani indices on nanostructures like carbon nanotube and boron nitride nanotube $[4,7]$.

Here we have considered finite, simple, and connected graphs. Consider a graph $G$ with a set of vertices and edges $V(G)$ and $E(G)$, respectively. The degree of a vertex $w$ is denoted by $d_{G}(w)$. The set of all vertices adjacent to $w$ are called the open neighborhood of $w$ and denoted by $N_{G}(w)$. The closed neighborhood of $w$ is the set $N_{G}[w]=N_{G}(w) \cup\{w\}$. The set $N_{G}[w]$ is the set of closed neighborhood vertices of $w$. Let $D_{G}(w)=d_{G}(w)+\sum_{v \in N_{G}(w)} d_{G}(w)$ be the degree sum of closed neighborhood vertices of $w$.

Definition 1.1. The first and second neighborhood Dakshayani indices are stated as

$$
N D_{1}(G)=\sum_{v w \in E(G)}\left[D_{G}(v)+D_{G}(w)\right], \quad N D_{2}(G)=\sum_{v w \in E(G)}\left[D_{G}(v) D_{G}(w)\right]
$$

Definition 1.2. The first and second hyper neighborhood Dakshayani indices are stated as

$$
H N D_{1}(G)=\sum_{v w \in E(G)}\left[D_{G}(v)+D_{G}(w)\right]^{2}, \quad H N D_{2}(G)=\sum_{v w \in E(G)}\left[D_{G}(v) D_{G}(w)\right]^{2}
$$

Definition 1.3. The Minus and Square neighborhood Dakshayani indices of a graph $G$ is stated as

$$
M N D(G)=\sum_{v w \in E(G)}\left|\left[D_{G}(v)-D_{G}(w) \mid\right], Q N D(G)=\sum_{v w \in E(G)}\right|\left[D_{G}(v)-D_{G}(w)\right]^{2} \mid
$$

Definition 1.4. The $F_{1}$ neighborhood Dakshayani index of a graph $G$ is stated as

$$
F_{1} N D(G)=\sum_{v w \in E(G)}\left[D_{G}(v)^{2}+D_{G}(w)^{2}\right]
$$

For the computation of topological indices on nanostructures, see $[1,2,12]$. In this paper, we carry forward the work done in [3] by using Neighborhood Dakshayani indices on carbon nanotube and Boron- nitride nanotube.

\section{Materials and Methods}

Our main results consist of degree-based neighborhood topological indices of carbon nanotubes and boron nitride nanotubes. To calculate the results, vertex partition, edge partition, analytical techniques, graph-theoretical tools, and a sum of degrees of neighbor methods are used.

\section{Results and Discussion}

\subsection{Boron-nitride graph.}

In inorganic chemistry, the boron-nitride graph is a simple, connected, and planar graph. The symbolic representation of boron-nitride is BN. The BN horizontal and vertical rings of are $p \times p .2 p^{2}+4 p$ is the order of graph, $O|G|$ and is as shown in Figure 2 and $3 p^{2}+4 p$ -1 is the size of the graph $E|G|$. The $2 \mathrm{D} \mathrm{BN}$ graph is classified as Cubic BN and Hexagonal $\mathrm{BN}$. In this proposed work, the hexagonal $\mathrm{BN}$ graph is considered. The BN consists of boron and nitrogen atoms, joined alternatively to form a hexagonal structure. The hexagons are formed such that an equal number of atoms of Boron (B) and nitrogen $(\mathrm{N})$ are involved in the formation. $0.145 \mathrm{~nm}$ is the bond-length between the atoms. The angle between BoronNitrogen-Boron or Nitrogen-Boron-Nitrogen is $120^{\circ}$. The hexagonal BN structure consists of a single layer and is found in nanotubes.

The edge classification based on the neighbor sum of the degrees of the end vertices of each edge of $B N(G)$ is denoted in Table 1. 


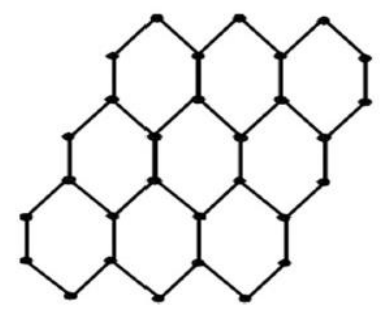

Figure 1. $3 \times 3$, hexagonal Boron nitride graph.

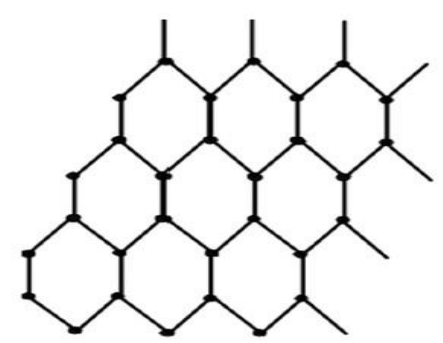

Figure 2. $p \times p$, hexagonal Boron nitride graph.

Table 1. The edge partition of $B N(G)$ For $p \geq 2$.

\begin{tabular}{l|l}
$E(v, w)$ & No. of Edges \\
Where $v w \in E(G)$ & 4 \\
\hline$E(4,5)$ & 2 \\
\hline$E(5,5)$ & 8 \\
\hline$E(5,7)$ & $8(p-2)$ \\
\hline$E(6,7)$ & $4(p-1)$ \\
\hline$E(7,9)$ & $(p-1)(3 p-5)$
\end{tabular}

Theorem 2.1. The first neighborhood Dakshayani index of hexagonal boron nitride nano-tube graph $\{B N T(G)\}$ for $p \geq 2$ is $54 p^{2}+24 p-34$

Proof.

$N D_{1}(G)=\sum_{v w \in E(G)}\left[D_{G}(v)+D_{G}(w)\right]$

$=E(4,5)(4+5)+E(5,5)(5+5)+E(5,7)(5+7)+E(6,7)(6+7)+E(7,9)(7+9)$

$+E(9,9)(9+9)$

$=4(9)+2(10)+8(12)+(8 p-16)(13)+(4 p-4)(16)+\left(3 p^{2}-8 p+5\right)(18)$

$=54 p^{2}+24 p-34$.

Theorem 2.2. The second neighborhood Dakshayani index of hexagonal boron nitride nanotube graph $\{B N T(G)\}$ for $p \geq 2$ is $243 p^{2}-60 p-109$

Proof.

$N D_{2}(G)=\sum_{v w \in E(G)}\left[D_{G}(v) D_{G}(w)\right]$

$=E(4,5)(4 \times 5)+E(5,5)(5 \times 5)+E(5,7)(5 \times 7)+E(6,7)(6 \times 7)+E(7,9)(7 \times 9)$

$+E(9,9)(9 \times 9)$

$=243 p^{2}-60 p-109$.

Theorem 2.3. The first hyper neighborhood Dakshayani index of hexagonal boron nitride nanotube graph $\{B N T(G)\}$ for $p \geq 2$ is $972 p^{2}-216 p-500$

Proof.

$H N D_{1}(G)=\sum_{v w \in E(G)}\left[D_{G}(v)+D_{G}(w)\right]^{2}$

$=E(4,5)(4+5)^{2}+E(5,5)(5+5)^{2}+E(5,7)(5+7)^{2}+E(6,7)(6+7)^{2}+E(7,9)(7+9)^{2}$

$+E(9,9)(9+9)^{2}$ 
$=972 p^{2}-216 p-500$.

Theorem 2.4. The first hyper neighborhood Dakshayani index of hexagonal boron nitride nano-tube graph $\{B N T(G)\}$ for $p \geq 2$ is $19683 p^{2}-22500 p+4355$

Proof.

$H N D_{2}(G)=\sum_{v w \in E(G)}\left[D_{G}(v) D_{G}(w)\right]^{2}$

$=E(4,5)(4 \times 5)^{2}+E(5,5)(5 \times 5)^{2}+E(5,7)(5 \times 7)^{2}+E(6,7)(6 \times 7)^{2}+E(7,9)(7 \times 9)^{2}$

$+E(9,9)(9 \times 9)^{2}$

$=19683 p^{2}-22500 p+4355$.

Theorem 2.5. The minus neighborhood Dakshayani index of hexagonal boron nitride nanotube graph $\{B N T(G)\}$ for $p \geq 2$ is $16 p-4$

Proof.

$\operatorname{MND}(G)=\sum_{v w \in E(G)}\left|\left[D_{G}(v)-D_{G}(w)\right]\right|$

$=E(4,5)|(4-5)|+E(5,5)|(5-5)|+E(5,7)|(5-7)|+E(6,7)|(6-7)|+E(7,9)|(7-9)|$

$+E(9,9)|(9-9)|$

$=16 p-4$.

Theorem 2.6. The square neighborhood Dakshayani index of hexagonal boron nitride nanotube graph $\{B N T(G)\}$ for $p \geq 2$ is $24 p+4$

Proof.

$Q N D(G)=\sum_{v w \in E(G)}\left|\left[D_{G}(v)-D_{G}(w)\right]^{2}\right|$

$=E(4,5)\left|(4-5)^{2}\right|+E(5,5)\left|(5-5)^{2}\right|+E(5,7)\left|(5-7)^{2}\right|+E(6,7)\left|(6-7)^{2}\right|+E(7,9)\left|(7-9)^{2}\right|$

$+E(9,9)\left|(9-9)^{2}\right|$

$=24 p+4$.

Theorem 2.7. The $F_{1}$ neighborhood Dakshayani index of hexagonal boron nitride nano-tube graph $\{B N T(G)\}$ for $p \geq 2$ is $486 p^{2}-96 p-214$

Proof.

$F_{1} N D(G)=\sum_{v w \in E(G)}\left[D_{G}(v)^{2}+\mathrm{D}_{\mathrm{G}}(w)^{2}\right]$

$=E(4,5)\left(4^{2}+5^{2}\right)+E(5,5)\left(5^{2}+5^{2}\right)+E(5,7)\left(5^{2}+7^{2}\right)+E(6,7)\left(6^{2}+7^{2}\right)+E(7,9)\left(7^{2}+9^{2}\right)$

$+E(9,9)\left(9^{2}+9^{2}\right)$

$=486 \mathrm{p}^{2}-96 \mathrm{p}-214$.

\subsection{Carbon nanotube graph.}

The carbon nanotube (CNT) graph is a simple, connected, and planar graph, as depicted in figure 3 and figure 4 . The CNT consists of two sets of rings viz. vertical and horizontal rings. The first one consists of $p$ vertical rings, and the other one consists of $p-1$ horizontal rings. It consists of $4 p^{2}+4 p-1$ vertices, and $6 p^{2}+3 p-2$ edges. The CNT's have a diameter approximately equal to $1 \mathrm{~nm}$. The structure of CNT affects the length and angle between the carbon atoms. In our work, we considered the $p \times p,(\mathrm{p}=\mathrm{q})$ rectangular section of the CNT graph for all $p \geq 3$.

The edge classification based on the neighbor sum of the degrees of the end vertices of each edge of $C N T(G)$ is denoted in Table 2. 


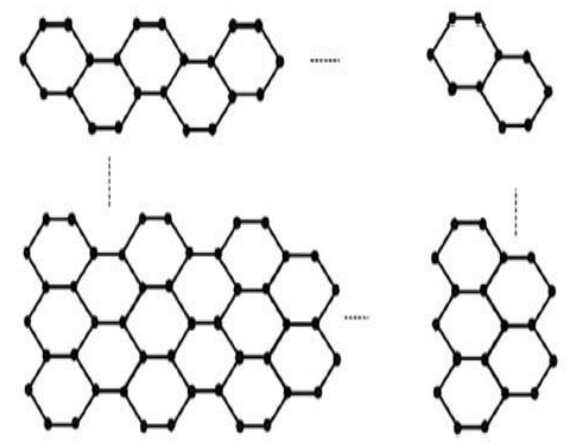

Figure 3. Carbon nanotube graph.

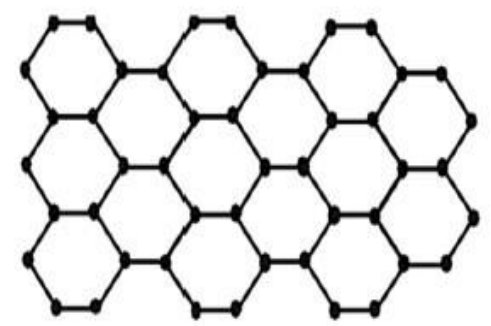

Figure 4. Carbon nanotube graph with $\mathrm{p}=3$.

Theorem 3.1. The first neighborhood Dakshayani index of Carbon nano-tube graph $\{C N T(G)\}$ for $p \geq 3$ is $108 p^{2}-14 p-40$

\section{Proof.}

$N D_{1}(G)=\sum_{v w \in E(G)}\left[D_{G}(v)+D_{G}(w)\right]$ $=E(4,5)(4+5)+E(5,5)(5+5)+E(5,7)(5+7)+E(5,8)(5+8)+E(6,7)(6+7)$

$+E(7,9)(7+9)$

$+E(8,8)(8+8)+E(8,9)(8+9)+E(9,9)(9+9)$

$=4(9)+2 p(10)+8(12)+(4 p-4)(13)+(4 p-10)(13)+(2 p-1)(16)+(2 p-2)(16)$

$+(4 p-4)(17)+\left(6 p^{2}-15 p+7\right)(18)$

$=108 p^{2}-14 p-40$.

Table 2. The edge partition of $C N T(G)$ For $p \geq 3$.

\begin{tabular}{l|l}
$E(v, w)$ & No. of Edges \\
Where $v w \in E(G)$ & 4 \\
\hline$E(4,5)$ & $2 p$ \\
\hline$E(5,5)$ & 8 \\
\hline$E(5,7)$ & $4(p-1)$ \\
\hline$E(5,8)$ & $2(2 p-5)$ \\
\hline$E(6,7)$ & $(2 p-1)$ \\
\hline$E(7,9)$ & $2(p-1)$ \\
\hline$E(8,8)$ & $4(p-1)$ \\
\hline$E(8,9)$ & $6 p^{2}-15 p+7$
\end{tabular}

Theorem 3.2. The second neighborhood Dakshayani index of Carbon nano-tube graph $\{C N T(G)\}$ for $p \geq 3$ is $486 p^{2}-295 p-132$

Proof.

$$
\begin{aligned}
& N D_{2}(G)=\sum_{v w \in E(G)}\left[D_{G}(v) D_{G}(w)\right] \\
& =E(4,5)(4 \times 5)+E(5,5)(5 \times 5)+E(5,7)(5 \times 7)+E(5,8)(5 \times 8)+E(6,7)(6 \times 7)+
\end{aligned}
$$

$E(7,9)(7 \times 9)$ 
$+E(8,8)(8 \times 8)+E(8,9)(8 \times 9)+E(9,9)(9 \times 9)$

$=486 p^{2}-295 p-132$.

Theorem 3.3. The first hyper neighborhood Dakshayani index of Carbon nano-tube graph $\{C N T(G)\}$ for $p \geq 3$ is $1944 p^{2}-112 p-546$

Proof.

$H N D_{1}(G)=\sum_{v w \in E(G)}\left[D_{G}(v)+D_{G}(w)\right]^{2}$

$=E(4,5)(4+5)^{2}+E(5,5)(5+5)^{2}+E(5,7)(5+7)^{2}+E(5,8)(5+8)^{2}+E(6,7)(6+7)^{2}$

$+E(7,9)(7+9)^{2}+E(8,8)(8+8)^{2}+E(8,9)(8+9)^{2}+E(9,9)(9+9)^{2}$

$=1944 p^{2}-112 p-546$.

Theorem 3.4. The first hyper neighborhood Dakshayani index of Carbon nano-tube graph $\{C N T(G)\}$ for $p \geq 3$ is $39366 p^{2}-46843 p+390$

Proof.

$H_{N D}(G)=\sum_{v w \in E(G)}\left[D_{G}(v) D_{G}(w)\right]^{2}$

$=E(4,5)(4 \times 5)^{2}+E(5,5)(5 \times 5)^{2}+E(5,7)(5 \times 7)^{2}+E(5,8)(5 \times 8)^{2}+E(6,7)(6 \times 7)^{2}$

$+E(7,9)(7 \times 9)^{2}+E(8,8)(8 \times 8)^{2}+E(8,9)(8 \times 9)^{2}+E(9,9)(9 \times 9)^{2}$

$=39366 p^{2}-46843 p+390$.

Theorem 3.5. The minus neighborhood Dakshayani index of Carbon nano-tube graph $\{C N T(G)\}$ for $p \geq 3$ is $12 p+10$

Proof.

$\operatorname{MND}(G)=\sum_{v w \in E(G)}\left|\left[D_{G}(v)-D_{G}(w)\right]\right|$

$=E(4,5)|(4-5)|+E(5,5)|(5-5)|+E(5,7)|(5-7)|+E(5,8)|(5-8)|+E(6,7)|(6-7)|$

$+E(7,9)|(7-9)|+E(8,8)|(8-8)|+E(8,9)|(8-9)|+E(9,9)|(9-9)|$

$=12 p+10$

Theorem 3.6. The square neighborhood Dakshayani index of Carbon nano-tube graph $\{C N T(G)\}$ for $p \geq 3$ is $16 p+24$

Proof.

$Q N D(G)=\sum_{v w \in E(G)}\left|\left[D_{G}(v)-D_{G}(w)\right]^{2}\right|$

$=E(4,5)\left|(4-5)^{2}\right|+E(5,5)\left|(5-5)^{2}\right|+E(5,7)\left|(5-7)^{2}\right|+E(5,8)\left|(5-8)^{2}\right|+E(6,7)\left|(6-7)^{2}\right|$

$+E(7,9)\left|(7-9)^{2}\right|+E(8,8)\left|(8-8)^{2}\right|+E(8,9)\left|(8-9)^{2}\right|+E(9,9)\left|(9-9)^{2}\right|$

$=16 p+24$.

Theorem 3.7. The first neighborhood Dakshayani index of Carbon nanotube graph $\{C N T(G)\}$ for $p \geq 3$ is $972 p^{2}-538 p-282$

Proof.

$F_{1} N D(G)=\sum_{v w \in E(G)}\left[D_{G}(v)^{2}+\mathrm{D}_{\mathrm{G}}(w)^{2}\right]$

$\left.+7^{2}\right)$

$=E(4,5)\left(4^{2}+5^{2}\right)+E(5,5)\left(5^{2}+5^{2}\right)+E(5,7)\left(5^{2}+7^{2}\right)+E(5,8)\left(5^{2}+8^{2}\right)+E(6,7)\left(6^{2}\right.$

$+E(7,9)\left(7^{2}+9^{2}\right)+E(8,8)\left(8^{2}+8^{2}\right)+E(8,9)\left(8^{2}+9^{2}\right)+E(9,9)\left(9^{2}+9^{2}\right)$

$=972 \mathrm{p}^{2}-538 \mathrm{p}-282$.

\section{Conclusions}

In the proposed work, we have calculated the values of Neighborhood Dakshyani indices for nanostructures such as hexagonal boron nitride nanotubes and carbon nanotubes. The results obtained are used to analyze the properties of nanotubes. Using Neighborhood Dakshyani indices to determine the properties of nanostructures includes various applications in biomedical, aerospace, water filtration, etc. 


\section{Funding}

This research received no external funding.

\section{Acknowledgments}

This research has no acknowledgment.

\section{Conflicts of Interest}

The authors declare no conflict of interest.

\section{References}

1. Aslam, A.; Ahmad, S.; Gao, W.J.Z.f.N.A. On certain topological indices of boron triangular nanotubes. 2017, 72, 711-716.

2. Akhter, S.; Imran, M. On molecular topological properties of benzenoid structures. Canadian Journal of Chemistry 2016, 94, 687-698, https://doi.org/10.1139/cjc-2016-0032.

3. Yousaf, A.; Alolaiyan, H.; Nadeem, M.; Razaq, A. Topological analysis of carbon and boron nitride nanotubes. Scientific Reports 2020, 10, 1-9, https://doi.org/10.1038/s41598-020-58372-1.

4. De, N. On Molecular Topological Properties of $\mathrm{TiO}_{2}$ Nanotubes. J. Nanosci. 2016, 1-5, https://doi.org/10.1155/2016\%2F1028031.

5. Gutman, I.; Polansky, O.E. Mathematical Concepts in Organic Chemistry. Springer, Berlin, 1986.

6. Hayat, S.; Imran, M. Computation of topological indices of certain networks. Applied Mathematics and Computation 2014, 240, 213-228, https://doi.org/10.1016/j.amc.2014.04.091.

7. Liu, J.-B.; Gao, W.; Siddiqui, M.K.; Farahani, M.R. Computing three topological indices for Titania nanotubes. AKCE International Journal of Graphs and Combinatorics 2016, 13, 255-260, https://doi.org/10.1016/j.akcej.2016.07.001.

8. Kulli, V.R. Neighbourhood Dakshayani indices. International Journal of Mathematical Archive 2019, 10, 23-31.

9. Kulli, V.R. Some Multiplicative Neighbourhood Dakshayani Indices of Certain Nanostructures. International Journal of Mathematics and its Applications 2019, 7, 209-217.

10. Kulli, V.R. $F_{1}$-Neighbourhood and square neighbourhood Dakshayani indices of some nano structures. International journal of engineering sciences and research technology 2019, 8, 126-138.

11. Mondal, S.; Bhosale, A.; De, N.; Pal, A. Topological properties of some nanostructures. Nanosystems: Physics, Chemistry, Mathematics 2020, 11, 14-24, https://doi.org/10.17586/2220-8054-2020-11-1-14-24.

12. Nadeem, M.; Yousaf, A.; Alolaiyan, H.; Razaq, A. Certain polynomials and related topological indices for the series of benzenoid graphs. Scientific Reports 2019, 9, 1-5, https://doi.org/10.1038/s41598-019-45721y.

13. Shanmukha, M.C.; Basavarajappa, N.S.; Anilkumar, K.N. Predicting physico-chemical properties of octane isomers using QSPR approach. Malaya Journal of Matematik 2020, 8, 104-116, https://doi.org/10.26637/MJM0801/0018.

14. Shanmukha, M.C.; Basavarajappa, N.S.; Shilpa, K.C.; Usha, A. Degree-based topological indices on anticancer drugs with QSPR analysis. Heliyon 2020, 6, 1-9, https://doi.org/10.1016/j.heliyon.2020.e04235.

15. Anil Kumar, K.N; Basavarajappa, N.S.; Shanmukha, M.C. QSPR analysis of Alkanes with certain degree based topological indices. Malaya Journal of Matematik 2020, 8, 314-330, https://doi.org/10.26637/MJM0802/0003.

16. Mondal, S.; De, N.; Pal, A. On Some New Neighborhood Degree-Based Indices for Some Oxide and Silicate Networks. J 2019, 2, 384-409, https://doi.org/10.3390/j2030026.

17. Trinajstic, N. Chemical Graph Theory. CRC Press, Boca Raton, FL. 1992. 